\title{
Tracking Single Dynamic MEG Dipole Sources Using the Projected Extended Kalman Filter
}

\author{
Yuchen Yao and A. Lee Swindlehurst
}

\begin{abstract}
This paper presents two new algorithms based on the Extended Kalman Filter (EKF) for tracking the parameters of single dynamic magnetoencephalography (MEG) dipole sources. We assume a dynamic MEG dipole source with possibly both time-varying location and dipole orientation. The standard EKF-based tracking algorithm performs well under the assumption that the dipole source components vary in time as a Gauss-Markov process, provided that the background noise is temporally stationary. We propose a Projected-EKF algorithm that is adapted to a more forgiving condition where the background noise is temporally nonstationary, as well as a Projected-GLS-EKF algorithm that works even more universally, when the dipole components vary arbitrarily from one sample to the next.
\end{abstract}

\section{INTRODUCTION}

Magnetoencephalography (MEG) is the measurement of magnetic fields resulting from the ensemble of neural activities in the human brain. It is a powerful non-invasive tool for investigating the mechanisms of brain-controlled physical movements, cognitive processes, and neurological and psychiatric disorders [1]. Researchers have obtained useful information by localizing the MEG signal sources related to such disorders and directly treated patients in the specific region of the brain that is abnormal. MEG signals are typically measured by an array of sensors, and the signals of interest are measured in the presence of spatially correlated interference due to the presence of background brain activity [2] [5] [6] [7].

Most prior work has focused on situations where the MEG sources and dipole moments are fixed. Some exceptions include [8], which studies sources with fixed locations but time-varying moments, and [9], where both are allowed to vary, but a separate estimation step is conducted at each time sample to determine the parameters. The standard Extended Kalman Filter (EKF) has been applied to tracking and estimating electroencephalography (EEG) and/or MEG dipole sources in [10] [11] [12]. The standard EKF works when the following assumptions are satisfied:

- Assumption I: the location of the MEG dipole source varies in time as a Gauss-Markov process;

- Assumption II: the dipole moment components of the MEG dipole source vary in time as a Gauss-Markov process;

- Assumption III: the interference is temporally stationary.

The authors are with the Department of Electrical Engineering and Computer Science, University of California, Irvine, CA 92697, USA yucheny@uci. edu
In this paper, we present two new EKF-based algorithms to relax the last two assumptions. The first approach is called the Projected-EKF, and it reduces the effect of temporally nonstationary noise by means of a denoising projection. The second algorithm couples a generalized least-squares (GLS) estimator of the dipole moments together with the ProjectedEKF to eliminate the need for Assumption II.

\section{DATA MODEL}

\section{A. Dipole Source Model}

The primary current distribution corresponding to an MEG signal source is modeled as an equivalent current dipole. A dipole source is modeled with a vector, comprised of the dipole moment in the $\mathrm{x}-, \mathrm{y}-$, and $\mathrm{z}$-directions. Let $\mathbf{p}_{s}$ be the dipole moment of the signal-of-interest (SOI): $\mathbf{p}_{s}(t)=$ $\left[p_{s_{x}}(t), p_{s_{y}}(t), p_{s_{z}}(t)\right]^{T}$. The location of the dipole source at time $t$ is defined by the three-dimensional rectangular coordinate $\left(x_{s}(t), y_{s}(t), z_{s}(t)\right)$. The location of the $j^{\text {th }}$ source of interference is defined by its coordinate $\left(x_{i_{j}}, y_{i_{j}}, z_{i_{j}}\right)$ with dipole moment $\mathbf{p}_{i_{j}}$.

\section{B. MEG Signal Model}

In the control state, the MEG signal received by each sensor only contains interference and background noise. In the task state, a stimulus-evoked SOI is generated and the signal received is the sum of the SOI and background noise. We assume a single SOI and $J$ sources of interference. The outputs from the $m^{t h}$ sensor at time $t$ in the two states are modeled as

$$
\begin{aligned}
& \text { Control State }\left(t \in T_{\text {control }}\right) \text { : } \\
& x_{m}(t)=\sum_{j=1}^{J} \mathbf{a}_{i_{m, j}} \mathbf{p}_{i_{j}}(t)+n_{m}(t) \\
& \text { Task State }\left(t \in T_{\text {task }}\right) \text { : } \\
& x_{m}(t)=\underbrace{\mathbf{a}_{s_{m}}(\beta(t)) \mathbf{p}_{s}(t)}_{\text {SOI term }}+\sum_{j=1}^{J} \mathbf{a}_{i_{m, j}} \mathbf{p}_{i_{j}}(t)+n_{m}(t)
\end{aligned}
$$

where $\mathbf{a}_{s, m}(\beta(t))$ and $\mathbf{a}_{i_{m, j}}$ are the respective $1 \times 3$ lead field vectors of the SOI and the $j^{\text {th }}$ interference source, $\beta(t)$ denotes the time-varying coordinate parameters $\left(x_{s}(t), y_{s}(t), z_{s}(t)\right)$, and $n_{m}(t)$ denotes the sensor noise. The outputs from the $M$ sensors in the control and task states are 
stacked in a column vector:

$$
\begin{aligned}
\mathbf{X}(t) & =\sum_{j=1}^{J} \mathbf{A}_{i_{j}} \mathbf{p}_{i_{j}}(t)+\mathbf{n}(t) \\
\mathbf{X}_{C}(t) & =\underbrace{\mathbf{A}_{s}(\beta(t)) \mathbf{p}_{s}(t)}_{\text {SOI term }}+\sum_{j=1}^{J} \mathbf{A}_{i_{j}} \mathbf{p}_{i_{j}}(t)+\mathbf{n}(t)
\end{aligned}
$$

\section{Three-component Vector Sensor}

It is well known that the radial component of a dipole source is not measurable using radially oriented sensors [2], because an MEG sensor can only receive signals that are orthogonal to its orientation. Here, we propose a threecomponent vector sensor in order to fully measure the magnetic field. The three-component vector sensor is nothing but a sensor group consisting of three separate sensors oriented orthogonally at the same location. Therefore, there are $3 M$ sensors distributed at $M$ sensor locations. In recent decades, extensive research has been conducted on the theory and design of vector sensors. Vector sensors have been applied for electromagnetic source localization and other electromagnetic-field applications [3] [4]. However, vector sensor for MEG measurements has not been developed yet.

\section{Model of the Dynamic SOI Dipole Source}

We define $\mathbf{s}(t)$ as the state vector of the SOI dipole source at time $t: \mathbf{s}(t)=\left[\mathbf{s}_{r}(t), \mathbf{p}_{s}^{T}(t)\right]^{T}$, where $\mathbf{s}_{r}(t)$ is $\left[x_{s}(t), y_{s}(t), z_{s}(t), v_{x_{s}}(t), v_{y_{s}}(t), v_{z_{s}(t)}\right]$, and $\left[v_{x_{s}}(t), v_{y_{s}}(t), v_{z_{s}(t)}\right]$ is the velocity of the SOI dipole source. Our task is to estimate this state vector at each time instant. A random walk model [13] on the velocities is used to model the motion of MEG SOI dipole sources. To keep the estimated motion within a reasonable region [14], we set up $K$ confining points on the imaginary human skull to generate a virtual potential field that will restrict its movement within the brain. The force generated by the $k^{\text {th }}$ confining point is written as a $3 \times 1$ vector:

$$
\mathbf{F}_{k}(t)=\frac{-F_{c r}}{d_{s}^{3}(\beta(t))}\left[\begin{array}{l}
x_{s}(t)-x_{c_{k}} \\
y_{s}(t)-y_{c_{k}} \\
z_{s}(t)-z_{c_{k}}
\end{array}\right],
$$

where $F_{c r}$ is a designed parameter that determines the overall strength of the repelling force, $d_{s}$ is the distance between the SOI dipole source and the $k^{t h}$ confining point, and $\left(x_{c_{k}}, y_{c_{k}}, z_{c_{k}}\right)$ is the coordinate of the $k^{t h}$ confining point. The resulting repelling force exerted on the SOI dipole source is the sum of the repelling forces from the $K$ confining points, denoted as $\mathbf{F}_{r}(t)=\left[F_{r_{x}}(t), F_{r_{y}}(t), F_{r_{z}}(t)\right]^{T}$.

Combining the random walk model and the repelling force yields the dynamic model of the SOI dipole source's location and velocity [14]:

$$
\mathbf{s}_{r}(t)=\boldsymbol{\Lambda} \mathbf{s}_{r}(t-1)+\left[\begin{array}{c}
0.5 \mathbf{F}_{r}(t-1) \\
\mathbf{F}_{r}(t-1)
\end{array}\right]+\left[\begin{array}{c}
\mathbf{0}_{3 \times 1} \\
\sigma_{v}(t)
\end{array}\right]
$$

where $\boldsymbol{\Lambda}=\left[\begin{array}{ll}\mathbf{I}_{3} & \mathbf{I}_{3} \\ \mathbf{0}_{3} & \mathbf{I}_{3}\end{array}\right]$ is a constant state transition matrix and $\sigma_{v}(t)=\left[\sigma_{x}(t), \sigma_{y}(t), \sigma_{z}(t)\right]$ is the Gaussian perturbation vector on the SOI dipole source velocity.
We also assume that the dipole components on the $\mathrm{x}$-, y-, z-directions satisfy the Gauss-Markov model, although this information is not exploited by the Projected-GLS-EKF algorithm:

$$
\mathbf{p}_{s}(t)=\mathbf{p}_{s}(t-1)+\sigma_{p}(t),
$$

where $\sigma_{p}(t)$ is the Gaussian perturbation vector on $\mathbf{p}_{s}(t-1)$.

\section{TRACKING ALGORITHMS}

\section{A. State-space Model}

Combining (5) and (6) yields the state dynamics equation

$$
\mathbf{s}(t)=\mathbf{f}(\mathbf{s}(t-1))+\mathbf{u}(t),
$$

which is a nonlinear function of $\mathbf{s}(t-1)$. The driving term is zero-mean, white Gaussian noise with known covariance $\mathbf{Q}$. The MEG measurement is written as a nonlinear function of $\mathbf{s}(t)$ :

$$
\mathbf{X}(t)=\mathbf{h}(\mathbf{s}(t))+\mathbf{w}(t),
$$

where $\mathbf{w}(t)$ is the background noise.

\section{B. Standard EKF}

The standard EKF algorithm requires all three of the assumptions put forth in the end of Section I. The background noise $\mathbf{w}(t)$ is assumed to be temporally stationary with a covariance matrix $\mathbf{W}$ estimated from the control state:

$$
\mathbf{W}=\frac{1}{N_{C}}\left\{\mathbf{X}_{C}-E\left[\mathbf{X}_{C}\right]\right\}\left\{\mathbf{X}_{C}-E\left[\mathbf{X}_{C}\right]\right\}^{T}
$$

where $\mathbf{X}_{C}$ is the control state data set and $N_{C}$ is the number of samples in the control state. The EKF also requires linearized versions of the state transition and observation functions:

$$
\begin{aligned}
\mathbf{G}_{t, t-1} & =\frac{\partial \mathbf{f}(\hat{\mathbf{s}}(t-1 \mid t-1))}{\partial \hat{\mathbf{s}}(t-1 \mid t-1)} \\
\mathbf{H}_{t, t-1} & =\frac{\partial \mathbf{h}(\hat{\mathbf{s}}(t \mid t-1))}{\partial \hat{\mathbf{s}}(t \mid t-1)}
\end{aligned}
$$

The standard EKF recursion is defined as follows:

$$
\begin{aligned}
\hat{\mathbf{s}}(t \mid t-1) & =\mathbf{f}(\hat{\mathbf{s}}(t-1 \mid t-1)) \\
\mathbf{R}_{t \mid t-1} & =\mathbf{G}_{t, t-1} \mathbf{R}_{t-1 \mid t-1} \mathbf{G}_{t, t-1}^{T}+\mathbf{Q} \\
\mathbf{K}_{t} & =\mathbf{R}_{t \mid t-1} \mathbf{H}_{t, t-1}^{T}\left[\mathbf{H}_{t, t-1} \mathbf{R}_{t \mid t-1} \mathbf{H}_{t, t-1}^{T}+\mathbf{W}\right]^{-1} \\
\hat{\mathbf{s}}(t \mid t) & =\hat{\mathbf{s}}(t \mid t-1)+\mathbf{K}_{t}[\mathbf{X}(t)-\mathbf{h}(\hat{\mathbf{s}}(t \mid t-1))] \\
\mathbf{R}_{t \mid t} & =\left(\mathbf{I}_{9}-\mathbf{K}_{t} \mathbf{H}_{t, t-1}\right) \mathbf{R}_{t \mid t-1},
\end{aligned}
$$

where $\mathbf{K}(t)$ is the Kalman gain, and $\mathbf{R}_{t \mid t-1}, \mathbf{R}_{t \mid t}$ are the predicted and filtered error covariance matrices.

\section{Proposed Algorithm 1: Projected-EKF}

If Assumption III does not hold, we are no longer able to estimate the covariance of the background noise by calculating $\mathbf{W}$. To address this problem, we choose to project out the interference prior to application of the EKF. To do this, we define $\hat{\mathbf{D}}$ to be the eigenvectors of $\mathbf{X}_{C}$ corresponding to the smallest eigenvalues. The choice of the dimension of $\hat{\mathbf{D}}$ is a design parameter whose choice can be based on standard techniques for model-order determination. 
Using $\hat{\mathbf{D}}$ to project the task state data set, we have:

$$
\begin{aligned}
\hat{\mathbf{D}}^{T} \mathbf{X}(t) & =\hat{\mathbf{D}}^{T} \mathbf{A}_{s}(\beta(t)) \mathbf{p}_{s}(t)+\sum_{j=1}^{J} \underbrace{\hat{\mathbf{D}}^{T} \mathbf{A}_{i} \mathbf{p}_{i}(t)}_{\approx 0}+\hat{\mathbf{D}}^{T} \mathbf{n}(t) \\
& \approx \hat{\mathbf{D}}^{T} \mathbf{A}_{s}(\beta(t)) \mathbf{p}_{s}(t)+\hat{\mathbf{D}}^{T} \mathbf{n}(t) .
\end{aligned}
$$

We then apply the EKF on this projected MEG measurement. The different forms of the Kalman gain matrix and the estimate update are:

$$
\begin{aligned}
\mathbf{K}_{t}= & \mathbf{R}_{t \mid t-1} \mathbf{H}_{t, t-1}^{T} \hat{\mathbf{D}}\left[\hat{\mathbf{D}}^{T} \mathbf{H}_{t, t-1} \mathbf{R}_{t \mid t-1} \mathbf{H}_{t, t-1}^{T} \hat{\mathbf{D}}\right. \\
& +\Sigma]^{-1} \\
\hat{\mathbf{s}}(t \mid t)= & \hat{\mathbf{s}}(t \mid t-1)+\mathbf{K}_{t} \hat{\mathbf{D}}^{T}[\mathbf{X}(t)-\mathbf{h}(\hat{\mathbf{s}}(t \mid t-1))] \\
\mathbf{R}_{t \mid t}= & \left(\mathbf{I}_{9}-\mathbf{K}_{t} \hat{\mathbf{D}}^{T} \mathbf{H}_{t, t-1}\right) \mathbf{R}_{t \mid t-1}
\end{aligned}
$$

where $\Sigma$ is the covariance of the projected $\mathbf{X}_{C}$. We refer to this algorithm as the Projected-EKF.

\section{Proposed Algorithm 2: Projected-GLS-EKF}

If the dynamic dipole moment model of Assumption II does not hold, then the above EKF approaches may not perform well. To overcome this problem, we present another algorithm here that exploits the fact that the data is linear in the dipole moments to estimate them at each time step.

After projecting out the interference, the following GLS cost function can be used to estimate $\mathbf{p}_{s}(t)$ directly:

$$
\begin{aligned}
C\left(\hat{\mathbf{p}}_{s_{t}}\right)= & \sum_{t=1}^{N}\left[\mathbf{X}(t)-\mathbf{A}_{s}(\beta(t)) \hat{\mathbf{p}}_{s_{t}}\right]^{T} \hat{\mathbf{D}} \Sigma^{-1} \hat{\mathbf{D}}^{T} \\
& \cdot\left[\mathbf{X}(t)-\mathbf{A}_{s}(\beta(t)) \hat{\mathbf{p}}_{s_{t}}\right] .
\end{aligned}
$$

The estimate of $\mathbf{p}_{s}(t)$ is easily obtained as

$$
\hat{\mathbf{p}}_{s_{t}}=\left[\mathbf{A}_{s}^{T}(\beta(t)) \Gamma \mathbf{A}_{s}(\beta(t))\right]^{-1} \mathbf{A}_{s}(\beta(t))^{T} \Gamma \mathbf{X}(t) .
$$

where $\Gamma=\hat{\mathbf{D}} \Sigma^{-1} \hat{\mathbf{D}}^{T}$. Because the components in the $\mathrm{x}-$, $\mathrm{y}-$, $\mathrm{z}$-directions are no longer treated as part of the state vector, the state vector is reduced to simply $\mathbf{s}_{r}(t)$.

The modified linearizations to the state transition and observation functions are

$$
\begin{aligned}
\mathbf{G}_{r_{t, t-1}} & =\frac{\partial \mathbf{f}_{r}\left(\hat{\mathbf{s}}_{r}(t-1 \mid t-1)\right)}{\partial \hat{\mathbf{s}}_{r}(t-1 \mid t-1)} \\
\mathbf{H}_{r_{t, t-1}} & =\frac{\partial \mathbf{h}_{r}\left(\hat{\mathbf{s}}_{r}(t \mid t-1), \hat{\mathbf{p}}_{s_{t \mid t-1}}\right)}{\partial \hat{\mathbf{s}}_{r}(t \mid t-1)},
\end{aligned}
$$

where $\mathbf{f}_{r}$ denotes the state transition function of $\mathbf{s}_{r}$, and $\mathbf{h}_{r}\left(\hat{\mathbf{s}}_{r}(t \mid t-1), \hat{\mathbf{p}}_{s_{t \mid t-1}}\right)$ equals

$$
\mathbf{A}_{s_{t \mid t-1}} \underbrace{\left[\mathbf{A}_{s_{t \mid t-1}}^{T} \Gamma \mathbf{A}_{s_{t \mid t-1}}\right]^{-1} \mathbf{A}_{s_{t \mid t-1}}^{T} \Gamma \mathbf{X}(t)}_{\hat{\mathbf{p}}_{s_{t \mid t-1}}} t \in T_{t a s k},
$$

where $\mathbf{A}_{s_{t \mid t-1}}$ denotes $\mathbf{A}_{s}(\beta(t \mid t-1))$, and $\beta(t \mid t-1)$ is the predicted SOI dipole source location. The Projected-GLS-
EKF algorithm is the implemented as follows:

$$
\begin{aligned}
& \hat{\mathbf{s}}_{r}(t \mid t-1)=\mathbf{f}_{r}\left(\hat{\mathbf{s}}_{r}(t-1 \mid t-1)\right) \\
& \mathbf{R}_{r_{t \mid t-1}}=\mathbf{G}_{r_{t, t-1}} \mathbf{R}_{r_{t-1 \mid t-1}} \mathbf{G}_{r_{t, t-1}}^{T}+\mathbf{Q}_{r} \\
& \hat{\mathbf{p}}_{s_{t \mid t-1}}=\left[\mathbf{A}_{s_{t \mid t-1}}^{T} \Gamma \mathbf{A}_{s_{t \mid t-1}}\right]^{-1} \mathbf{A}_{s_{t \mid t-1}}^{T} \Gamma \mathbf{X}(t) \\
& \mathbf{K}_{t}=\mathbf{R}_{r_{t \mid t-1}} \mathbf{H}_{r_{t, t-1}}^{T} \hat{\mathbf{D}} \\
& \cdot\left[\hat{\mathbf{D}}^{T} \mathbf{H}_{r_{t, t-1}} \mathbf{R}_{r_{t \mid t-1}} \mathbf{H}_{r_{t, t-1}}^{T} \hat{\mathbf{D}}+\Sigma\right]^{-1} \\
& \hat{\mathbf{s}}_{r}(t \mid t)=\hat{\mathbf{s}}_{r}(t \mid t-1)+\mathbf{K}_{t} \hat{\mathbf{D}}^{T}\left[\mathbf{X}(t)-\mathbf{h}_{r}(t \mid t-1)\right] \\
& \mathbf{R}_{r_{t \mid t}}=\left(\mathbf{I}_{6}-\mathbf{K}_{t} \hat{\mathbf{D}}^{T} \mathbf{H}_{r_{t, t-1}}\right) \mathbf{R}_{r_{t \mid t-1}} \\
& \hat{\mathbf{p}}_{s_{t \mid t}}=\left[\mathbf{A}_{s_{t \mid t}}^{T} \Gamma \mathbf{A}_{s_{t \mid t}}\right]^{-1} \mathbf{A}_{s_{t \mid t}}^{T} \Gamma \mathbf{X}(t),
\end{aligned}
$$

where $\mathbf{Q}_{r}$ is the covariance of perturbation on $s_{r}(t)$, and $\mathbf{h}_{r}\left(\hat{\mathbf{s}}(t \mid t-1), \hat{\mathbf{p}}_{s_{t \mid t-1}}\right)$ is replaced with $\mathbf{h}_{r}(t \mid t-1)$ for simplicity.

\section{SIMULATION RESULTS}

In our simulation, an 180-channel (60 vector sensors) MEG measurement system is employed. The number of interference sources is set to 4 , and they are randomly distributed on the surface of the brain. The control and task states both have 200 samples. We evaluate the performance of the three algorithms (two proposed algorithms plus the regular EKF) by simulating the tracking and estimation process in three different simulated scenarios. In these scenarios, the designed Signal to Interference plus Noise Ratio (SINR) is as low as $0 \mathrm{~dB}$.

In Scenario I, the MEG background noise is temporally stationary. The position, velocity, and dipole components of the SOI dipole source all vary according to the state-space model defined in Section III. Fig. 1 shows the tracking results of the three algorithms. There is no significant difference in the performance of these algorithms. In Scenario II, the MEG background noise is temporally nonstationary, and the interference changes from the control state to the task state. The position, velocity, and the SOI dipole components of the SOI source also vary according to the state-space model. Fig. 2 shows the tracking results of the three algorithms in Scenario II, in which the performance of regular EKF is inferior to the other two algorithms. In Scenario III, we keep the nonstationary background noise in Scenario II, and the position and the velocity of the SOI dipole source vary according to (5). However, instead of obeying the dynamic model, the dipole components vary randomly from sample to sample. The standard EKF fails in this scenario, and while the Projected-EKF works for some period of time, we see in Fig. 3 that it eventually diverges from the true track. On the other hand, the Projected-GLS-EKF works throughout the entire data collection.

\section{CONCLUSIONS}

We have proposed two new algorithms based on the EKF to track single dynamic MEG dipole sources. The first algorithm combines the EKF with a projection that uses control-state data to filter out the background noise prior to application of the EKF. This helps eliminate the effect of 


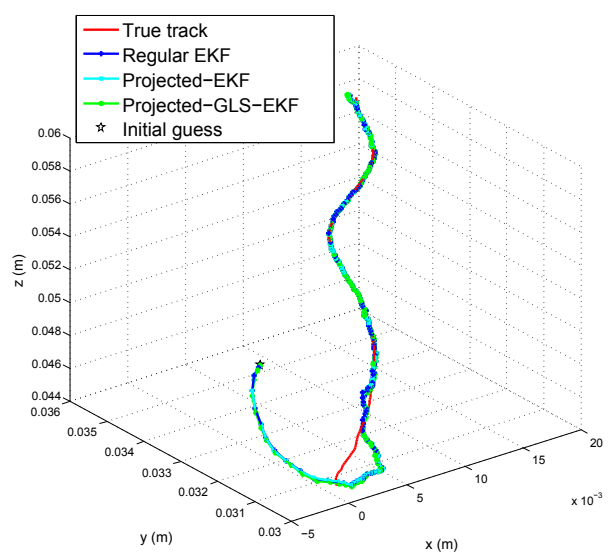

Fig. 1. Tracking results in Scenario I

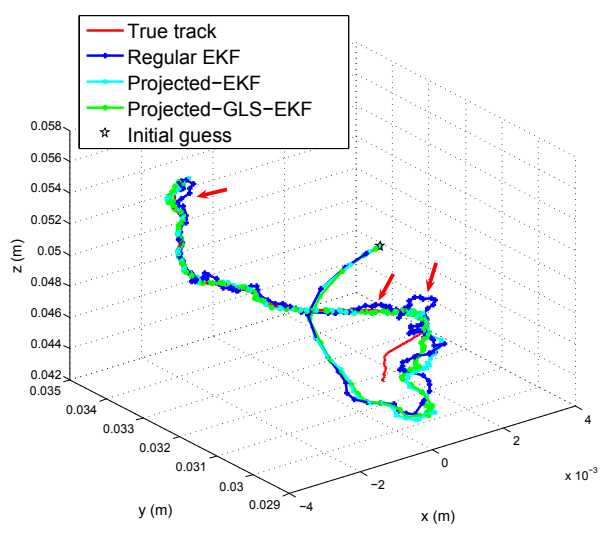

Fig. 2. Tracking results in Scenario II

any nonstationarity present in the data before it can impact the tracking performance. The second algorithm exploits the fact that the model depends linearly on the dipole moment, allowing the moment parameters to be estimated in closedform at each time sample. This reduces the dimension of the state space, and removes any requirement for modeling the time evolution of the dipole moment. Our simulation results demonstrate the benefits associated with these two algorithms. However, when the dipole components of the SOI satisfy (6), the projected-EKF is slightly better than the GLS-Projected-EKF since the GLS method does not exploit the Gauss-Markov model.

\section{ACKNOWLEDGMENTS}

The authors appreciate Dr. Jianqi Wang's insightful suggestions on this research project. Yuchen Yao thanks the UC Irvine Block Fellowship for grant support.

\section{REFERENCES}

[1] A. Nehorai and A. Dogandžić, "Estimation of Propagating Dipole Sources by EEG/MEG Sensor Arrays", Proceedings of the 32nd Asilomar Conference on Signals, Systems and Computers, Pacific Grove, CA, November, 1998.

[2] J. C. Mosher, P. S. Lewis, and R. M. Leahy, "Multiple Dipole Modeling and Localization from Spatio-Temporal MEG Data," IEEE Transactions on Biomedical Engineering, vol. 39, no. 6, pp 541-557, 1992.

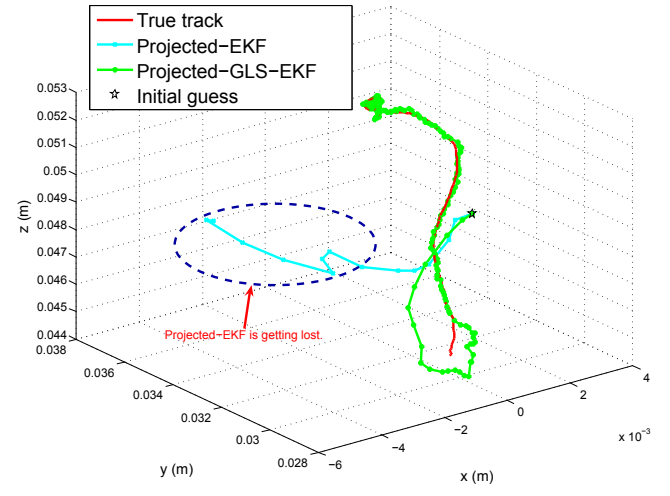

Fig. 3. Tracking results in Scenario III

[3] A. Nehorai and E. Paldi, "Vector-sensor array processing for electromagnetic source localization," IEEE Transactions on Signal Processing, vol. 42, no. 2, pp 376-398, 1994.

[4] S. Kordic, P. J. A. Munter, "Three-dimensional magnetic-field sensors," IEEE Transactions on Electron Devices, vol. 35, no. 6, pp 771-779, 1988.

[5] K Sekihara, K. E. Hild, II, S. S. Dalal, and S. S. Nagarajan, "Performance of Prewhitening Beamforming in MEG Dual Experimental Conditions," IEEE Transactions on Biomedical Engineering, vol. 55, no. 3, pp 1112-1121, 2008.

[6] B. D. van Veen, W. van Drongelen, M. Yuchtman, and A. Suzuki, "Localization of Brain Electrical Activity via Linearly Constrained Minimum Variance Spatial Filtering," IEEE Transaction on Biomedical Engineering, vol. 44, pp. 867-880, 1997.

[7] S. C. Wu, A. L. Swindlehurst, and Y. C. Yao, "Direct Interference Suppression in EEG/MEG Dipole Source Localization," Proceedings of the International Conference on Acoustics, Speech and Signal Processing 2009, pp. 574-577, Dallas, TX, March, 2009.

[8] M. Scherg and D. Cramon, "Evoked dipole source potentials of the human auditory cortex," Electroencephalography and Clinical Neurophysiology, vol. 65, pp. 344-360, 1986.

[9] A. Dogandžić, and A. Nehorai, "Estimating Evoked Dipole Responses in Unknown Spatially Correlated Noise with EEG/MEG Arrays," IEEE Transaction on Signal Processing, vol. 48, no. 1, pp. 13-25, 2000.

[10] E. Somersalo, A. Voutilainen and J.P. Kaipio, "Non-stationary magnetoencephalography by Bayesian filtering of dipole models," Inverse Problems, vol. 19, pp 1047-1063, 2003.

[11] J. Antelis and J. Minguez, "Dynamic Solution to the EEG Source Localization Problem Using Kalman Filters and Particle Filters," Proceedings of the Annual International Conference of the IEEE Engineering in Medicine and Biology Society, 2009, pp. 77-80, Minneapolis, MN, September, 2009.

[12] J. Antelis and J. Minguez, "DYNAMO: Dynamic MultiModel Source Localization Method for EEG and/or MEG," Proceedings of the Annual International Conference of the IEEE Engineering in Medicine and Biology Society, 2010, pp. 5141-5144, Buenos Aires, Argentina, September, 2010.

[13] X. Li and V. Jilkov, "Survey of Maneuvering Target TrackingPart I: Dynamic models," IEEE Transaction on Aerospace and Electronic Systems, vol. 39, no. 4, pp. 1333-1364, 2003.

[14] A. S. Paul and E. A. Wan, "RSSI-Based Indoor Localization and Tracking Using Sigma-Point Kalman Smoothers," IEEE Journal of Selected Topics in Signal Processing, vol. 3, no. 5, pp. 860-873, 2009. 